\title{
New clinical pathways for keratoconus
}

DM Gore, AJ Shortt and BD Allan

\begin{abstract}
Pre-2000, the clinical management of keratoconus centred on rigid contact lens fitting when spectacle corrected acuity was no longer adequate, and transplantation where contact lens wear failed. Over the last decade, outcome data have accumulated for new interventions including corneal collagen crosslinking, intracorneal ring implantation, topographic phototherapeutic keratectomy, and phakic intraocular lens implantation. We review the current evidence base for these interventions and their place in new management pathways for keratoconus under two key headings: corneal shape stabilisation and visual rehabilitation. Eye (2013) 27, 329-339; doi:10.1038/eye.2012.257; published online 21 December 2012

Keywords: review; keratoconus; crosslinking; intracorneal ring; intraocular lens; meta-analysis
\end{abstract}

\section{Conventional keratoconus management}

For the age group 10-44 years, the prevalence of keratoconus in white Europeans has been estimated 1 in 1750, rising to 1 in 450 in South Asians. ${ }^{1}$ Until 2000, disease progression in keratoconus was managed with spectacles until irregular astigmatism necessitated rigid contact lens fitting, then corneal transplantation where contact lenses failed. No interventions were available to arrest or slow disease progression, and corneal transplantation was required in up to $21 \%$ of keratoconic eyes. ${ }^{2}$ Using established techniques (Markov modelling), the lifetime cost of conventional keratoconus treatment has been estimated at US\$25168 per patient, with the likelihood of primary and repeat corneal transplantation being the key cost determinants. ${ }^{3}$ Given the relatively high prevalence of keratoconus and the frequency with which corneal transplantation is required, this represents a significant health economic burden. ${ }^{3}$

While penetrating keratoplasty (PK) for keratoconus generally achieves good visual outcomes, long-term graft survival in keratoconic eyes declines rapidly after the second decade. Primary graft survival rates from the Australian Graft Registry are $89 \%$ at 10 years, $49 \%$ at 20 years, and $17 \%$ at 23 years. ${ }^{4}$ These rates fall further with repeat grafts. Ten-year survival rates are $53 \%$ for second and $33 \%$ for third grafts. ${ }^{5}$ Most grafts for keratoconus are performed in relatively young patients. In an effort to reduce the rate of repeat transplantation and associated complications, many surgeons now use deep anterior lamellar keratoplasty (DALK), with preservation of the host endothelium, as the transplantation procedure of choice in keratoconus. Accelerated long-term endothelial cell loss observed after PK does not occur after DALK. ${ }^{6}$ Extrapolation from observed endothelial cell loss rates predicts a median graft survival of 49 years for DALK vs 17 years for PK. ${ }^{7}$ But current DALK techniques are technically challenging. Intraoperative complications and conversion to PK are common. A recent UK transplant registry comparison of results for PK and DALK in keratoconus showed a high early failure rate for DALK. ${ }^{8}$ This was attributed to the learning curve in the surgical transition from PK to DALK for many surgeons in the period studied (1999-2005). Where transplantation is successful, irregular astigmatism and high postoperative anisometropia commonly prevent successful visual rehabilitation in spectacles without recourse to further surgical intervention. ${ }^{4}$

Corneal transplantation is an effective but costly intervention in advanced keratoconus (stage IV modified Krumeich classification, ${ }^{9}$ anterior $3 \mathrm{~mm}$ zone steep keratometry $[\mathrm{K}]$ $>$ 55D; Table 1), with an estimated incremental cost-utility ratio of between $\$ 1942^{10}$ and $\$ 3025^{11}$ per quality-adjusted lifeyear. The life impact of repeat intervention, and slow, often incomplete, visual rehabilitation after transplantation should not be underestimated. ${ }^{12}$ Transplantation costs, and evidence for reduced quality of life in contact lens wearers, ${ }^{13}$
Moorfields Eye Hospital, London, UK

Correspondence: DM Gore, External Disease Service, Moorfields Eye Hospital, 162 City Road, London EC1V 2PD, UK. Tel: +44 (0)20 7566 2320; Fax: +44 (0)207566 2019 . E-mail: dan.gore@ moorfields.nhs.uk

Received: 12 September 2012

Accepted in revised form: 31 October 2012 Published online: 21 December 2012 
Table 1 Modified Krumeich classification of keratoconus ${ }^{9}$

\begin{tabular}{ll}
\hline Stage & Characteristics \\
\hline I & Eccentric corneal steepening \\
& Induced myopia and/or astigmatism $<5 \mathrm{D}$ \\
& Corneal radii $\leq 48 \mathrm{D}$ \\
& Vogt's striae, no scars \\
& Induced myopia and/or astigmatism $>5 \mathrm{D},<8 \mathrm{D}$ \\
& Corneal radii $\leq 53 \mathrm{D}$ \\
II & No central scars \\
& Corneal thickness $\geq 400 \mu \mathrm{m}$ \\
& Induced myopia and /or astigmatism $>8 \mathrm{D},<10 \mathrm{D}$ \\
& Corneal radii $>53 \mathrm{D}$ \\
III $\quad$ No central scars \\
& Corneal thickness $200-400 \mu \mathrm{m}$ \\
& Refraction not measurable \\
& Corneal radii $>55 \mathrm{D}$ \\
IV $\quad$ Central scars, perforation \\
& Corneal thickness $<200 \mu \mathrm{m}$ \\
& D,
\end{tabular}

Abbreviation: $\mathrm{D}$, dioptres.

Note that on a Pentacam scan result, K1 represents corneal curvature in the flat central $3 \mathrm{~mm}$ zone, $\mathrm{K} 2$ represents the steep curvature in the $3 \mathrm{~mm}$ zone; Kmax (denoted by the white diamond symbol) represents the steepest point over the entire scanned anterior corneal surface. Thresholds for progression used in contemporary studies (Table 2) refer to Kmax (or Kmin), as opposed to the above classification which is equivalent to the 3-mm zone $\mathrm{K} 2$

underline the need for a new, more proactive approach to keratoconus management.

\section{New pathways for keratoconus management}

Over the last decade, outcome data have accumulated for new interventions in keratoconus which promise to reduce transplantation rates significantly, arrest disease progression, and save many patients from long-term reliance on rigid contact lens wear. These interventions include corneal collagen crosslinking (CXL), intracorneal ring implantation (ICRS), topographic photorefractive keratectomy (topoPRK), and phakic intraocular lens (pIOL) implantation. They all require that ocular surface inflammation (eg, atopic keratoconjunctivitis) associated with keratoconus is well controlled before treatment, and none is applicable to advanced (stage IV) disease with corneal scarring. Beyond these basic common requirements, definition of the indications, timing and sequence for new interventions in keratoconus continues to evolve. Based on the current evidence base, we outline new proactive pathways for keratoconus management addressing two essential aims: shape stabilisation and visual rehabilitation.

\section{Methodology}

We searched MEDLINE (1950-2012) and the Cochrane Central Register of Controlled Trials using the following search terms: keratoconus; corneal crosslinking; crosslinkage; intracorneal ring segment; topoPRK; and pIOL. For the meta-analysis, two authors (DG and AS) independently assessed the search results for randomised-controlled trials (RCTs) of corneal crosslinking in keratoconus. The authors of one $\mathrm{RCT}^{14}$ were contacted for clarification of one trial methodology and they provided additional unpublished data. A risk of bias assessment was performed on studies and only studies with a low risk of selection, performance, detection, and attrition bias were included. Data extraction was done independently by DG and AS. Data synthesis was performed using a meta-analysis software package, Review Manager (RevMan, The Nordic Cochrane Centre, The Cochrane Collaboration, Copenhagen, 2008). The effect of the intervention was expressed as odds ratios and confidence intervals (CIs) were calculated. The Mantel-Haenszel fixed-effect method of meta-analysis was used.

\section{Defining disease progression-monitoring keratoconus}

Defining disease progression and the threshold for surgical intervention in keratoconus patients under observation remains a challenge. Assuming no diurnal variation in corneal shape, the coefficient of repeatability (CoR) should correspond closely to the limits of agreement for successive measures of corneal tomography indices, ${ }^{15}$ defining a threshold beyond which there is a $95 \%$ probability that an observed difference reflects disease progression rather than measurement inaccuracy. Keratometry measurement repeatability in keratoconic eyes (stage II, $3 \mathrm{~mm}$ zone steep $K \leq 53 \mathrm{D}$ ) has been shown to be significantly poorer than in normal corneas, with a 1D CoR for steepest keratometry (Kmax) using Fourier-domain anteriorsegment OCT (Casia SS-1000 Tomey Corp., Nagoya, Japan) and 1.5D using a Scheimpflug-based imaging device (Pentacam HR, Oculus GmbH, Wetzlar, Germany). ${ }^{16}$ This compares with CoR values in normal corneas of $<0.5 \mathrm{D}$ for both imaging devices. ${ }^{16}$ Based on this, a change in Kmax of $+1.5 \mathrm{D}$ vs measurement at presentation on Pentacam image comparison maps may be a useful contemporary threshold indication for CXL using a widely available corneal tomography device. A lower threshold ( $+1 \mathrm{D}$ vs baseline $\mathrm{Kmax}$ ) is used in contemporary studies (Table 2). Changes in other criteria used to define disease progression (Table 2) may tip the balance in favour of intervention where the observed increase in Kmax is $<+1.5 \mathrm{D}$.

The frequency with which patients should be screened and the optimum corneal tomography method also require additional research. After diagnosis, we are currently monitoring six monthly for 2 years with annual review subsequently. More frequent initial monitoring 
may be valuable in younger patients at high risk of rapid progression. Where possible, rigid contact lenses should be left out for a minimum of 2 weeks before each topography examination to reduce corneal warpage.

\section{Shape stabilisation}

\section{Standard crosslinking}

Corneal collagen crosslinking (CXL) using the standard, epithelium-off protocol originally described by Wollensak et $a l^{17}$ is effective in arresting disease progression (52\% stabilizing, 45\% regressing) with 3\% of eyes progressing despite treatment. ${ }^{14,18}$ Long-term outcomes (mean 52 months follow-up, range 48-60 months) for standard CXL have been reported in the Siena Eye Cross Study, ${ }^{19}$ a prospective, non-randomised study of 44 eyes with progressive keratoconus treated with CXL and fellow eye controls. Control eyes, in which disease progression was less rapid than treated eyes before assignment, were observed initially then crossed over to CXL after 2 years. Their data confirmed a halt in

Table 2 Criteria for keratoconus progression vs baseline measurement used in contemporary clinical trials of corneal collagen crosslinking 14,18,21

\section{Kmax $\geq 1 \mathrm{D}$ increase}

Kmax - Kmin $\geq 1 \mathrm{D}$ increase

Kmean $\geq 0.75 D$ increase

Pachymetry $\geq 2 \%$ decrease in CCT

Corneal apex power $>1 \mathrm{D}$ increase

MRSE $>0.5 \mathrm{D}$

Abbreviations: CCT, central corneal thickness; D, dioptres; Kmax, steepest keratometry; Kmin, flattest keratometry; MRSE, manifest refractive spherical equivalent.

Kmean $=(\mathrm{Kmax}+\mathrm{Kmin}) / 2$; corneal apex power is measured with cone location and magnitude index (CLMI). ectatic progression in all treated eyes, with a mean reduction in Kmax of 2 dioptres (D), while fellow eyes progressed by a mean 1.5D for 24 months (before crossover) before also demonstrating comparable reductions in Kmax after CXL. Refraction stabilised at 2 years and spectacle corrected distance visual acuity (CDVA) improved $2 \pm 1$ Snellen lines (mean \pm SD). ${ }^{19}$ Twoyear outcome data from an uncontrolled prospective study $^{20}$ in 40 children (mean age $14.2 \pm 1.7$ years) with mild disease ( $3 \mathrm{~mm}$ zone steep $K \leq 53 \mathrm{D}$ ) confirmed similar findings with both UDVA and CDVA improving by two lines (logMAR) and a reduction in Kmax of 1.27D.

Further evidence for the efficacy of standard, epithelium-off CXL is found in three RCTs with fellow eye controls that have published 12-month results. ${ }^{14,18,21}$ Excluding one study ${ }^{18}$ in which control eyes were crossed over to treatment after just 3 months, we combined 1-year post-CXL keratometric and acuity outcome data for patients $(n=31)$ in the remaining two trials. Clinically significant ectatic progression (increase in $\mathrm{Kmax} \geq 1 \mathrm{D}$ at 12 months) was observed in $32 \%$ untreated control eyes and 3\% eyes treated with CXL ( $P=0.01$, z-test); whereas significant regression (decrease in $\mathrm{Kmax} \geq 1 \mathrm{D}$ at 12 months) was seen in $45 \%$ of CXL treated eyes and 10\% untreated control eyes $(P=0.005$, z-test) (Figures 1a and b). After conversion of 12 month acuity data to $\log$ MAR values, combined data showed a statistically significant improvement in CDVA for patients treated with CXL ( $P=0.04$, z-test).

Spontaneous disease regression does not normally occur in keratoconus, and apparent disease regression (decrease in $\mathrm{Kmax} \geq 1 \mathrm{D}$ ) in $10 \%$ of control eyes in RCTs may simply reflect limitations in measurement accuracy for contemporary topographers ${ }^{16}$ — a problem compounded by corneal warpage in rigid contact lens

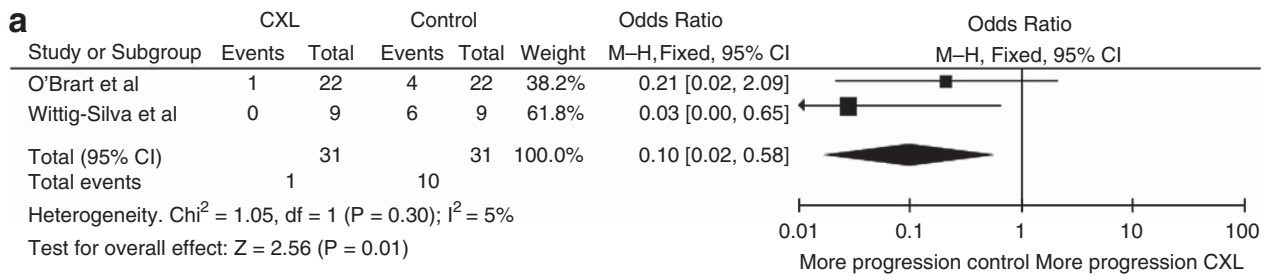

\begin{tabular}{|c|c|c|c|c|c|c|c|c|c|c|}
\hline \multirow{2}{*}{ S Study or Subgroup } & \multicolumn{2}{|c|}{ Experimental } & \multicolumn{2}{|c|}{ Control } & \multicolumn{3}{|c|}{ Odds Ratio } & \multirow{2}{*}{\multicolumn{2}{|c|}{$\begin{array}{c}\text { Odds Ratio } \\
\text { MàH, Fixed, 95\% Cl }\end{array}$}} & \\
\hline & Events & Total & Events & Total & Weight & $\mathrm{M}-\mathrm{H}$ & Fixed, $95 \% \mathrm{Cl}$ & & & \\
\hline O'Brart et al & 9 & 22 & 3 & 22 & $88.7 \%$ & 4.38 & {$[0.99,19.36]$} & & & \\
\hline Wittig -Silva et al & 5 & 9 & 0 & 9 & $11.3 \%$ & 23.22 & {$[1.04,517.93]$} & & & \\
\hline Total $(95 \% \mathrm{Cl})$ & & 31 & & 31 & $100.0 \%$ & 6.51 & {$[1.78,23.78]$} & & & \\
\hline Total events & 14 & & 3 & & & & & & & \\
\hline \multicolumn{5}{|c|}{ Heterogeneity. $\mathrm{Chi}^{2}=0.92, \mathrm{df}=1(\mathrm{P}=0.34) ; \mathrm{I}^{2}=0 \%$} & & & 0.001 & 0.1 & 10 & 1000 \\
\hline \multicolumn{5}{|c|}{ Test for overall effect: $Z=2.83(P=0.005)$} & & & Mor & sion c & trol More re & CXL \\
\hline
\end{tabular}

Figure 1 Forest plot of meta-analysis of 12 month results from placebo-controlled randomised trials showing (a) disease progression (defined in these trials by an increase in maximum keratometry $(K \max ) \geq 1 \mathrm{D}$ ) is significantly less likely after collagen CXL; and (b) disease regression (reduction of Kmax $\geq 1 \mathrm{D}$ ) is significantly more likely after CXL. 
wearers. These limitations and varying inclusion criteria (Table 2) in contemporary studies underline the problem of defining disease progression in keratoconus and defining thresholds for intervention.

Although generally safe, $14,18,19,21,22$ a range of complications is seen after standard, epithelium-off CXL. Sterile corneal infiltrates, thought to be an immune reaction to bacterial antigen deposition, were observed in $7.6 \%$ of patients in a prospective series of 117 eyes with 12 month follow-up after CXL reported by Koller et al. ${ }^{22}$ Culture-proven bacterial keratitis with permanent loss of vision has also been reported. ${ }^{23}$ Mild stromal haze is common after CXL and typically resolves within a year without detriment to visual acuity; but mid-stromal scarring was observed in $3 \%$ of cases by Koller et al, ${ }^{22}$ and $3 \%$ (95\% CI 1-9\%) lost two or more lines of Snellen CDVA. Although the mechanism of visual loss was not identified, risk factors for loss of vision in this study were age $>35$ years and pre-operative CDVA $>20 / 25$.

\section{Keratocyte and endothelial cell toxicity}

Keratocyte apoptosis has been observed at up to $350 \mu \mathrm{m}$ depth in experimental and clinical studies of the standard CXL protocol in which UVA light at $370 \mathrm{~nm}$ is applied at an irradiance of $3 \mathrm{~mW} / \mathrm{cm}^{2}$ for $30 \mathrm{~min}^{.24}$ Rates of endothelial cell loss similar to background age-related change were recorded in the Siena Cross Study ${ }^{19}$ where treated corneas had $\geqslant 400 \mu \mathrm{m}$ central thickness after epithelial removal, but significant cell loss and corneal endothelial failure have been reported in thinner corneas..$^{25,26}$ Shielding by riboflavin saturation in a $400-\mu \mathrm{m}$-thick cornea reduces irradiance at the endothelial level to $0.18 \mathrm{~mW} / \mathrm{cm}^{2},{ }^{24}$ well below the cytotoxic threshold of $0.35 \mathrm{~mW} / \mathrm{cm}^{2}$ previously established in porcine endothelial cell cultures. ${ }^{27}$ Hypotonic riboflavin is now commonly used to increase corneal thickness before UVA exposure in thinner corneas. This technique was originally described by Hafezi et al. ${ }^{28}$ They reported no complications or progression of ectasia at 6 months in a series of 20 eyes with $>320 \mu \mathrm{m}$ initial corneal thickness after epithelial removal. Similar encouraging 1 year results for CXL using hypotonic riboflavin in thinner corneas were reported by Raiskup $e a^{29}$ in a prospective series of 32 eyes.

\section{Rapid crosslinking}

Rapid treatment protocols for CXL based on shorter UVA exposure times and higher irradiances are emerging. The underlying premise in rapid CXL is that delivering a similar total energy over a shorter period of time will not compromise safety or efficacy in comparison with the standard protocol $\left(30 \mathrm{~min}\right.$ at $\left.3 \mathrm{~m} \mathrm{~W} / \mathrm{cm}^{2}\right)$. Stress-strain measurements on porcine corneal strips treated using a rapid protocol $\left(9 \mathrm{~min}\right.$ at $\left.10 \mathrm{~mW} / \mathrm{cm}^{2}\right)$ demonstrated equivalent increases in corneal stiffness in comparison with the standard protocol..$^{30}$ In a recent prospective randomised trial, ${ }^{31}$ contralateral eyes of 21 patients with progressive keratoconus were randomised to either conventional or rapid CXL $\left(7 \mathrm{~mW} / \mathrm{cm}^{2}\right.$ for $\left.15 \mathrm{~min}\right)$. A halt in ectasia progression was seen in all cases at a mean follow-up of 46 months. Both groups responded similarly: CDVA improved from 20/60 to 20/40, UDVA from $20 / 30$ to $20 / 25$, mean sphere reduced by $2.4 \mathrm{D}$ and Kmax reduced from 49.5D to 46.1D. Endothelial cell loss was less in the rapid CXL group $\left(-100\right.$ cells $\left./ \mathrm{mm}^{2}\right)$ than in the conventional CXL group $\left(-250\right.$ cells $\left./ \mathrm{mm}^{2}\right)$.

\section{Transepithelial crosslinking}

Transepithelial CXL, in which riboflavin is delivered using enhancers of epithelial permeability rather than epithelial debridement, promises to deliver the benefits of standard epithelium-off treatments without the painful rehabilitation and complications of epithelial removal. Epithelial-toxic agents used include benzalkonium chloride (BAC), sodium ethylenediaminetetraacetic acid (EDTA), tetracaine, proparacaine, ethanol, and gentamicin. A non-toxic reversible synthetic non-selective ion channel-forming peptide, NC-1059, has also been shown in a chick corneal model to enhance riboflavin permeability across an intact epithelium. ${ }^{32}$ Two prospective, paired-eye studies in humans have documented small, but significant improvements in CDVA over fellow untreated eyes at 12 and 18 months, respectively, with a trend towards deterioration in topographic and acuity measures in non-treated control eyes. ${ }^{33,34}$ Improvements in corneal curvature ( $\sim 2 \mathrm{D}$ mean reduction in Kmax) were similar to those seen after epithelium-off CXL. Statistically significant improvements in CDVA were also observed in an uncontrolled prospective study, ${ }^{35}$ though the authors recorded conflicting keratometric results depending on the imaging device used (progression by Scheimpflug, no change by Placido disc). In addition to drug-induced epithelial disruption, Stojanovic et $a l^{36}$ described the use of a riboflavin-soaked polyvinyl acetal pledget (Merocel sponge, Medtronic, Inc., Minneapolis, MN, USA) inserted into the conjunctival sac to produce microabrasions of the superficial epithelial layers with eyelid blinking. In conjunction with proparacaine, gentamicin, and BAC, their 12-month results showed significant improvements in UDVA, CDVA, refraction, and keratometric indices.

Optical coherence tomography (OCT) imaging suggests that crosslinking may be concentrated in the anterior corneal stroma after transepithelial $\mathrm{CXL},{ }^{34}$ with a demarcation line typically visible at around $100 \mu \mathrm{m}$ 
depth as opposed to $\sim 250 \mu \mathrm{m}$ after epithelium-off CXL. Transepithelial and standard, epithelium-off CXL may, therefore, be complimentary. Filippello et al ${ }^{34}$ argue that since crosslinking appears to be concentrated at different stromal levels for the two modalities, transepithelial treatment could be used where epithelium-off CXL fails. Conversely, provided further clinical evidence continues to support comparable efficacy and enhanced safety $v$ s standard, epithelium-off CXL, we believe that transepithelial CXL could have an important first-line treatment role in shape stabilisation for newly diagnosed keratoconus, with the back-up of epithelium-off CXL if ectatic progression still occurs (Figure 2).

Until now, the conventional approach has been to intervene with CXL only for patients with documented disease progression. Factors predictive of an increased risk of disease progression in keratoconus include young age ( $\leq 35$ years), steep keratometry, high astigmatism, reduced CDVA (irregular astigmatism), ethnicity other than white European, and documented progression in the contralateral eye. ${ }^{37-39}$ These risk factors for disease progression are often present at presentation, and may be compounded if further disease progression is allowed to occur. Based on this, the proven efficacy of CXL, 14,18,19,21,22 the higher risk of CXL-related visual loss aged $>35$ years, ${ }^{22}$ and the relative safety of transepithelial treatment, ${ }^{33,34}$ we believe that there is a rational argument for transepithelial CXL at presentation for patients $\leq 35$ years of age with keratometric stage II disease (Figures 2 and 3). ${ }^{9}$ For patients over 35 years without other risk factors, the risk of progression is lower (and CXL-related complications higher), so no intervention is required at presentation.

\section{Initial intervention in keratoconus}

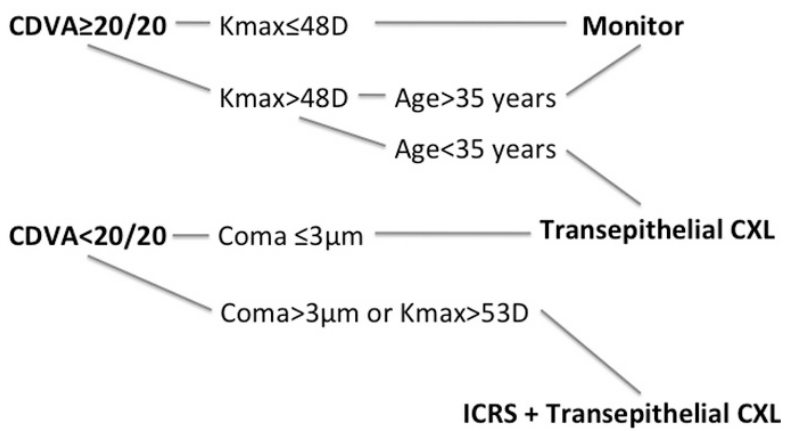

Figure 2 A decision tree for intervention at presentation in keratoconus. We are currently exploring the role for transepithelial collagen CXL at presentation for younger patients with keratometric stage II disease; and ICRS in combination with transepithelial CXL to provide a gross shape correction in patients with reduced spectacle CDVA at presentation and higher levels of coma or keratometric stage III disease.
As transepithelial CXL protocols continue to develop, further clinical trials documenting safety and efficacy are required to develop the evidence base for early intervention strategies.

\section{Visual rehabilitation}

In the future, early intervention with CXL should greatly reduce the numbers of patients dependent on rigid contact lenses and corneal transplantation for visual rehabilitation in keratoconus. For the present, many patients are already past the point at which they still have good spectacle corrected or unaided acuity. Current strategies for contact lens fitting in keratoconus are comprehensively described by Barnett and Mannis, ${ }^{40}$ and summarised briefly below. While transplantation remains the principal treatment for contact lens intolerant patients with stage IV keratoconus, newer interventions can be combined for earlier stage disease with the aim of restoring good spectacle corrected or unaided vision.

\section{Contact lens fitting}

Soft lenses and soft toric lenses can provide good visual rehabilitation in early keratoconus; but RGP lenses are generally required to neutralise significant corneal surface irregularity. RGP lenses provide good tear exchange, but lens stability deteriorates as the corneal profile steepens in more advanced keratoconus.

Traditional corneal RGP lenses are between 8 and $10 \mathrm{~mm}$ in diameter. Newer intralimbal RGP lenses (10.5-12 mm diameter) can improve corneal coverage and centration in some patients, ${ }^{41}$ but the increased diameter may make application and removal more difficult. 'Piggyback'

\section{Shape Stabilisation in Keratoconus}

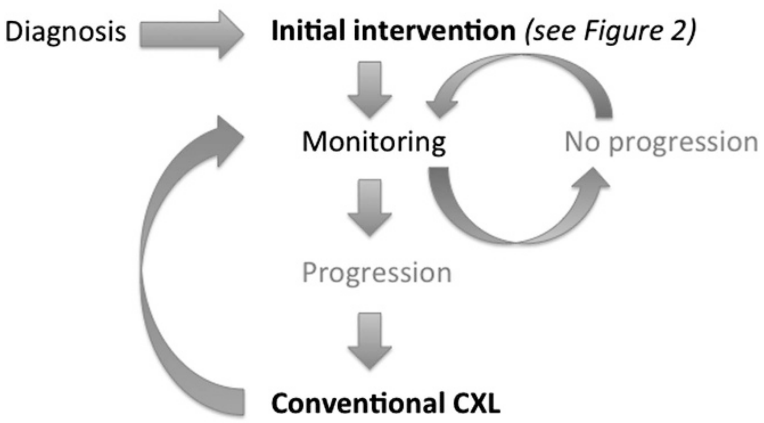

Figure 3 A pathway for shape stabilisation after initial intervention in keratoconus. Emerging transepithelial corneal CXL protocols avoid most of the complications associated with epithelium-off CXL but may be less effective. ${ }^{33,34}$ CXL can be repeated if there is continued disease progression. 
(soft beneath RGP) lens combinations and hybrid (RGP centre and a soft skirt) lenses can improve wearing time in patients with poor RGP lens tolerance. Where these options fail, RGP scleral (and semi-scleral) lenses, which vault clear of the corneal surface, are a solution applicable to almost any corneal shape. ${ }^{42}$ Scleral lenses can rescue vision in patients with late stage diseaseparticularly where technical obstacles such as a thin peripheral cornea limit options for corneal transplantation.

Despite advances in contact lens fitting for keratoconus, contact lens dependence may be associated with significant impairment of vision-related quality of life that continues to decline over time. ${ }^{13}$

\section{Gross corneal shape adjustment-intracorneal ring segments}

Intrastromal corneal ring segments (ICRS) are effective in flattening the corneal shape and improving vision for most recipients with keratoconus, but the magnitude of effect is highly variable; and inclusion of different implantation techniques, different types of ICRS, and patients with late stage disease in earlier studies ${ }^{43-46}$ makes the existing evidence base for ICRS harder to interpret.

Many surgeons now use smaller diameter ICRS designs (Table 3) and femtosecond laser assisted implantation. Earlier case series reporting manual implantation of the original Intacs ICRS (designed for simple myopic rather than keratoconus correction) are therefore not considered here. Femtosecond laser channel cutting has, by ensuring a consistent cut depth, both facilitated and enhanced safety in ICRS implantation. ${ }^{47}$ Flattening of central curvature with ICRS implantation increases with smaller ring diameter and greater segment thickness. The penalty for using a smaller diameter ring is a greater potential for dysphotopsia. The triangular profile used in Ferrara and Keraring ICRS is designed to reduce forward light scatter by total internal reflection of incident light, but further clinical studies are required to quantify dysphotopsia-associated with ICRS implantation.

In a series with 12-month postoperative results of 50 consecutive femtosecond laser Keraring ICRS cases reported by Coskunseven et al, ${ }^{48}$ approximately two-thirds of patients gained spectacle CDVA compared with pre-implantation levels (1-4 Snellen lines). Only two patients lost two lines of spectacle CDVA, and neither wanted the implants removed because of gains in UDVA. Overall improvement in mean keratometry was $\sim 3 \mathrm{D}(50.6 \pm 4 \mathrm{D}$ pre-ICRS; $47.6 \pm 4.5 \mathrm{D}$ post-ICRS) with larger gains for cases with a steeper initial shape (stage III, $3 \mathrm{~mm}$ zone steep $K>53 \mathrm{D}$ ) in which thicker ICRS was implanted. Asymmetric ICRS implantation (asymmetric arc length and thickness in superior and inferior ICRS) or single ring implantation can be used in patients with reduced spectacle CDVA associated with high preoperative coma (cone decentration). Results in a smaller series of 21 cases of femtosecond laser Keraring ICRS implantation reported by Shabayek and Alio ${ }^{49}$ were similar, with significant gains in overall higher order aberration levels for patients with high starting levels of coma (>3 microns). A retrospective series ${ }^{50}$ of 173 femtosecond laser cut eyes comparing Intacs SK and Keraring SI6 (both with a $6 \mathrm{~mm}$ diameter optical zone) observed comparable improvements in vision and keratometry, with no statistically significant differences between the two ICRS models.

Femtosecond ICRS implantation has a good safety profile. A recent multicenter retrospective review ${ }^{47}$ of 850 eyes with femtosecond Keraring ICRS implantation using an Intralase femtosecond laser for ring channel creation reported a $5.7 \%$ overall complication rate.

Table 3 Characteristics of available intracorneal ring segments (ICRS)

\begin{tabular}{|c|c|c|c|c|c|c|}
\hline Name & Manufacturer & $\begin{array}{c}\text { Internal } \\
\text { diameter } / \mathrm{mm}\end{array}$ & $\begin{array}{c}\text { External } \\
\text { diameter } / \mathrm{mm}\end{array}$ & $\begin{array}{l}\text { Arc length/ } \\
\text { degree }\end{array}$ & Profile & Thickness/mm \\
\hline Intacs & $\begin{array}{l}\text { Addition Technology Inc. Sunnyvale, } \\
\text { CA, USA }\end{array}$ & 6.8 & 8.1 & 150 & Hexagonal & $\begin{array}{l}0.25-0.45 \text { (0.05 } \\
\text { increments) }\end{array}$ \\
\hline Intacs SK & $\begin{array}{l}\text { Addition Technology Inc. Sunnyvale, } \\
\text { CA, USA }\end{array}$ & 6.0 & 7.3 & 150 & Oval & 0.4 and 0.45 \\
\hline Ferrara & $\begin{array}{l}\text { Mediphacos Ltd, } \\
\text { Belo Horizonte, Brazil }\end{array}$ & 4.4 & 5.6 & 160 & Triangular & $0.2-0.35$ \\
\hline $\begin{array}{l}\text { Kerrarings } \\
\text { SI5 }\end{array}$ & $\begin{array}{l}\text { Mediphacos Ltd, } \\
\text { Belo Horizonte, Brazil }\end{array}$ & 5.0 & - & $\begin{array}{c}90,120 \\
160, \text { and } 210\end{array}$ & Triangular & $0.15-0.35$ \\
\hline $\begin{array}{l}\text { Kerrarings } \\
\text { SI6 }\end{array}$ & $\begin{array}{l}\text { Mediphacos Ltd, } \\
\text { Belo Horizonte, Brazil }\end{array}$ & $5.5,6.0$ & - & $\begin{array}{c}90,120 \\
150, \text { and } 210\end{array}$ & Triangular & $0.15-0.35$ \\
\hline MyoRing & $\begin{array}{l}\text { Dioptex } \mathrm{GmbH} \\
\text { Linz, Austria }\end{array}$ & $5-8$ & - & 360 & Triangular $^{a}$ & $\begin{array}{l}0.2-0.4(0.02 \\
\text { increments })\end{array}$ \\
\hline
\end{tabular}

${ }^{\text {a }}$ Convex anteriorly and concave posteriorly. 
Intraoperative complications did not result in failure to complete ICRS implantation. Incomplete ring channel formation was the commonest complication (2.7\%). In these cases, ring channels can be completed manually allowing ICRS implantation to proceed. Endothelial perforation was evident in $0.6 \%$ cases. In this circumstance, channel creation $90 \mu \mathrm{m}$ superficial to the initial attempted depth can be completed 1 month after surgery. The target depth for femtosecond Keraring implantation is $80 \%$ of corneal thickness at the location of the ring channel ( $5 \mathrm{~mm}$ diameter). Ultrasound

pachymetry was used in this study. Availability of more accurate regional pachymetry methods, OCT in particular, may eliminate the risk of perforation. Postoperative complications occurred in $1.6 \%$ of cases. Within-channel migration of ring segments was noted in seven eyes and treated successfully with repositioning and a sutured vertical incision closure. Four cases of stromal thinning over the ICRS and two cases of corneal melting required implant removal. As with endothelial perforation, melting was attributed to inaccurate regional pachymetry and superficial channel creation in relatively thin corneas. Infection (an infiltrate at the entry site) was seen in one case in this series and treated without ICRS explantation. Problems for this study are lack of acuity, refractive, and keratometric outcome data. The authors also do not state the follow-up period studied or whether these were consecutive cases. Nonetheless, data from this and smaller prospective series ${ }^{47,49}$ indicate that sightthreatening complications are rare.

Very little long-term data are available for ICRS, and any effect of ICRS implantation on disease progression remains uncertain. In a case series of Intacs ICRS with 3-year follow-up in 13 eyes, significant increases in average $K$ values were observed between 6 months and 3 years, indicating that disease stabilisation was not achieved by ICRS alone. ${ }^{51}$

\section{Combined ICRS and CXL}

ICRS implantation can be combined with CXL, ${ }^{52-56}$ but the treatment sequence is important: ICRS appears more effective in improving corneal shape before the cornea is stiffened with CXL. Coskunseven et al, ${ }^{52}$ in an RCT comparing CXL first and ICRS later (group 1) with ICRS first and later CXL (group 2), showed significantly greater improvements (mean 7 months follow-up) in spectacle CDVA (three line gain (group 1); two line gain (group 2), $P<0.01$ ) and manifest astigmatism (2.48D mean absolute cylinder reduction (group 1); $1.76 \mathrm{D}$ reduction (group 2), $P<0.05$ ) where ICRS was performed first. Simultaneous CXL and ICRS may be equally effective. El-Raggal showed a trend towards greater improvement at 12 months in mean $K$ values where simultaneous ICRS and CXL was performed vs CXL 6 months after ICRS $(50.2 \pm 3.8 \mathrm{D}$ to $44.9 \pm 2.9 \mathrm{D}$ simultaneous treatment; $50.4 \pm 3.8 \mathrm{D}$ to $47.3 \pm 3.5 \mathrm{D}$ delayed $\mathrm{CXL}, P=0.046$ ), although there were no differences in UDVA, CDVA, or refractive error. ${ }^{54}$ But this study was underpowered ( $n=8$ each arm). More evidence is required to determine the optimum interval between ICRS and CXL in combined treatment; but the practical advantages to combining ICRS with transepithelial treatment in particular are clear. An interesting variation here has been described by Saelens et $a l^{53}$ who augmented stromal riboflavin penetration by injection into the ring channels before transepithelial CXL.

\section{ICRS implantation protocols}

With increasing availability of femtosecond laser technology, OCT regional pachymetry, and intraoperative guidance systems to help reduce axial misplacement during implantation, ICRS implantation should become safer and more predictable. Several important gaps in the evidence base for this intervention exist, but there are already strong arguments for ICRS implantation for gross corneal shape correction in keratoconus patients with reduced CDVA-particularly if they are contact lens intolerant. Disease progression after standard, epithelium-off CXL is more likely for patients with later stage III disease ( $3 \mathrm{~mm}$ zone steep $K>53 \mathrm{D}){ }^{22}$ and most patients with stage III keratometric changes already have reduced CDVA. Because contemporary ICRS implantation has a good safety profile and results are better if implantation is performed simultaneously with or before CXL, the balance of existing evidence suggests a role for ICRS implantation in combination with transepithelial CXL at presentation for patients with keratometric stage III keratoconus, or for earlier stage disease if vision is reduced (spectacle CDVA $\leq 20 / 25)$ in association with high levels of coma $(>3 \mu \mathrm{m})$ (Figure 2$)$.

Inclusion criteria in Coskunseven's recent prospective series of femtosecond Keraring ICRS implantation include a minimum central corneal thickness of 350 and $450 \mu \mathrm{m}$ at the incision site. ${ }^{48}$ No upper limit for keratometry values was specified. Neovascularisation has not been reported after femtosecond Keraring ICRS implantation and $5 \mathrm{~mm}$ diameter ICRS are within the block of tissue normally removed in any subsequent transplantation procedure. Where corneal thickness is adequate and there is no central scar, it is probably reasonable to consider ICRS implantation with CXL in patients with advanced stage III disease since there should be no increased risk of failure in any subsequent corneal transplant. But a stratified analysis of patients with contact lens fitting problems associated with steep 
keratometry is required to determine whether ICRS is a viable alternative to corneal transplantation for this group or whether there is an upper limit to the Kmax for ICRS. For the present, limits for ICRS implantation are probably best defined by Coskunseven's pachymetric inclusion criteria above. ${ }^{48}$

\section{Thermal keratoplasty}

Microwave thermal keratoplasty is being explored as a possible alternative to ICRS and transplantation for gross corneal shape correction in advanced keratoconus. Although large initial shape improvements can be obtained, almost complete regression is seen within a year. Kato et al ${ }^{57}$ treated 21 advanced keratoconic eyes with topography guided conductive keratoplasty (with intraoperative keratometric monitoring): mean keratometry values were $55 \pm 8 \mathrm{D}$ at baseline, $45 \pm 9 \mathrm{D}$ at 1 week, $50 \pm 7 \mathrm{D}$ at 1 month, and $54 \pm 7 \mathrm{D}$ at 3 months. Studies are in progress to determine the extent to which shape regression after thermokeratoplasty can be modulated by combination with CXL.

\section{Fine corneal shape adjustment-PRK}

Combination with CXL may allow safe application of surface excimer laser ablation techniques to fine tune corneal shape in keratoconus. To date, topographyguided PRK has been studied in combined therapy. ${ }^{58-60}$ But wavefront ablation would be a viable alternative in cases with good aberrometry data.

For simultaneous combined treatment, in which CXL is applied immediately after PRK, the aim is to improve CDVA by reducing irregular astigmatism rather than to correct spherocylindrical error fully. Reasons for this are the requirement for a $400-\mu \mathrm{m}$ residual stromal bed after ablation to allow safe CXL, and the danger of hypermetropic overcorrection if spherical equivalent reductions induced by CXL are not anticipated. Simultaneous combined treatment has clear advantages for patient comfort (the epithelium is only removed once), but takes no account of improvements in corneal regularity and spectacle CDVA that might normally be produced by CXL alone.

In a large comparative case series with minimum 2-year postoperative follow-up, Kanellopoulos ${ }^{60}$ compared outcomes of 198 eyes treated with topography-guided PRK followed immediately by CXL (simultaneous combined treatment) with an earlier series of 127 eyes treated with topography-guided PRK a minimum of 6 months after CXL (sequential combined treatment). Simultaneous combined treatment produced greater improvement across a range of measures: LogMAR CDVA improved from $0.39 \pm 0.3 \mathrm{D}$ to
$0.11 \pm 0.2 \mathrm{D}$, with a reduction in spherical equivalent of $-3.2 \pm 1.4 \mathrm{D}$ and mean keratometry of $-3.5 \pm 1.3 \mathrm{D}$.

This compares with the sequential group's CDVA improvement from $0.41 \pm 0.3 \mathrm{D}$ to $0.16 \pm 0.2 \mathrm{D}$, spherical equivalent reduction of $-2.5 \pm 1.2 \mathrm{D}$ and mean keratometry reduction of $-2.75 \pm 1.3 \mathrm{D}$. Haze scores were also significantly better for simultaneous combined treatment. In all, $20 \mathrm{~s}$ intraoperative applications of mitomycin $\mathrm{C}$ were used throughout, and the maximum ablation depth was limited to $50 \mu \mathrm{m}$. The essential problem for this comparison is that the time interval between CXL and PRK for sequential treatment was not specified, and may have been considerably shorter than the 2-year period in which refractive results typically continue to improve after CXL alone. ${ }^{19}$ Further study is required to quantify any gain in spectacle CDVA over CXL alone for simultaneous combined CXL and PRK, and to determine whether increased haze scores for sequential combined treatment observed by Kanellopoulos are still evident where PRK is performed later in the post-CXL wound healing cycle, a minimum of 2 years after treatment.

The current evidence base does not allow a clear recommendation with regard to the place for simultaneous combined CXL and PRK. But topographyguided or wavefront-driven PRK is a reasonable option to choose once refraction is stable after CXL if spectacle CDVA remains poor (Figure 4).

\section{Visual Rehabilitation in Keratoconus}

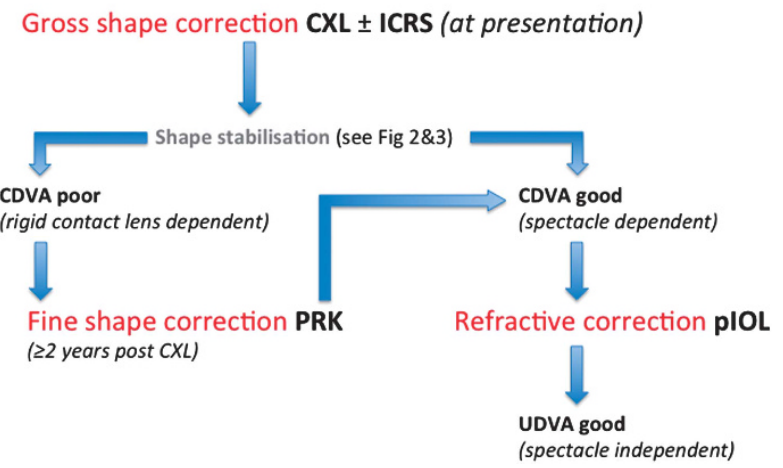

Figure 4 A pathway for visual rehabilitation in stage II and III keratoconus. Initial intervention in keratoconus (Figure 2) may include collagen $\mathrm{CXL} \pm$ intracorneal ring segment implantation (ICRS). Neither intervention provides a predictable shape change. After a 2-year period to allow shape stabilisation postCXL, 19,76 further fine shape correction with topographic PRK $^{58-60}$ may therefore be required to achieve good spectacle CDVA. If CDVA is good but uncorrected distance vision remains poor (UDVA) remains poor, then patients may opt for pIOL implantation ${ }^{61-66}$ to complete visual rehabilitation. 


\section{Refractive correction-pIOL implantation}

For patients with a stable corneal shape and good CDVA, pIOL implantation can be used to complete functional visual rehabilitation. A number of recent papers have reported effective refractive correction using pIOLs in keratoconus (post-operative spherical equivalent range $-0.08 \pm 0.4$ to $+0.1 \pm 0.4 ; 64-67 \%$ within $0.5 \mathrm{D}$ and $84-100 \%$ within 1D of target refraction) ${ }^{61-66}$ and for post-keratoplasty ametropia. ${ }^{67}$ Of the pIOLs available, ${ }^{68}$ the Visian ICL (Staar Surgical, Monrovia, CA, USA) offers the longest safety track record for an injectable pIOL and is available in a wide range of powers (including toric correction up to 6D). Pesando et al ${ }^{69}$ reported retrospective 10 -year follow-up data in $59 \mathrm{ICL}$ implanted hypermetropic eyes, showing a mean endothelial cell loss of $4.7 \%$, mostly occurring within the first few weeks of implantation and remaining almost unchanged thereafter. Edelhauser et $a l^{70}$ reported prospective, multicenter, 4-year follow-up data on 526 eyes with a $2-3 \%$ cell loss rate over the first 3 years, followed by a cell increase of $0.1 \%$ between years 3 and 4 , suggesting that endothelial remodelling and stability may have been achieved.

Combined ICRS and pIOL implantation has been described, ${ }^{71-74}$ but refractive correction with pIOLs is highly predictable, ${ }^{75}$ whereas the refractive effect of ICRS implantation is highly variable. ${ }^{43-46,52-56}$ We would therefore argue for gross shape correction with ICRS implantation followed by CXL, fine shape correction with PRK if necessary, then finally pIOL implantation as a logical pathway to visual rehabilitation in grade II-III keratoconus (Figure 4).

\section{Summary}

Thick case note files documenting the progression of keratoconus towards corneal transplantation may soon be a historical anecdote among ophthalmologists. While recent advances in contact lens technology have benefited many patients, the last few years have also seen new and exciting developments in both stabilizing and reversing ectasia, with interventions for visual rehabilitation originally developed in refractive surgery finding new applications in keratoconus. The clinical pathways we outline here attempt to clarify the positioning of new interventions in the treatment of keratoconus with reference to an incomplete evidence base. Clinical pathways for keratoconus will continue to evolve with further innovation and the guidance of future research.

\section{Conflict of interest}

The authors declare no conflict of interest.

\section{Acknowledgements}

This work was supported in part by the UK National Institute for Health Research Biomedical Research Centre in Ophthalmology at Moorfields Eye Hospital and UCL Institute of Ophthalmology.

\section{References}

1 Pearson A, Soneji B, Sarvananthan N. Does ethnic origin influence the incidence or severity of keratoconus? Eye 2000; 14: $625-628$

2 Lass JH, Lembach RG, Park SB, Hom DL, Fritz ME, Svilar GM et al. Clinical management of keratoconus. A multicenter analysis. Ophthalmology 1990; 97(4): 433-445.

3 Rebenitsch RL, Kymes SM, Walline JJ, Gordon MO. The lifetime economic burden of keratoconus: a decision analysis using a Markov model. Am J Ophthalmol 2011; 151(5): 768-773.

4 Kelly TL, Williams KA, Coster DJ. for the Australian Corneal Graft Registry. Corneal transplantation for keratoconus: a registry study. Arch Ophthalmol 2011; 129(6): 691-697.

5 Kelly TL, Coster DJ, Williams KA. Repeat penetrating corneal transplantation in patients with keratoconus. Ophthalmology 2011; 118(8): 1538-1542.

6 Reinhart WJ, Musch DC, Jacobs DS, Lee WB, Kaufman SC, Shtein RM. Deep anterior lamellar keratoplasty as an alternative to penetrating keratoplasty. Ophthalmology 2011; 118(1): 209-218.

7 Borderie VM, Sandali O, Bullet J, Gaujoux T, Touzeau O, Laroche L. Long-term results of deep anterior lamellar versus penetrating keratoplasty. Ophthalmology 2012; 119(2): 249-255.

8 Jones MNA, Armitage WJ, Ayliffe W, Larkin DF, Kaye SB. on behalf of the NHSBT Ocular Tissue Advisory Group and Contributing Ophthalmologists (OTAG Audit Study 5). Penetrating and deep anterior lamellar keratoplasty for keratoconus: a comparison of graft outcomes in the United Kingdom. Invest Ophthalmol Vis Sci 2009; 50(12): 5625-5629.

9 Colin J, Velou S. Current surgical options for keratoconus. J Cataract Refract Surg 2003; 29(2): 379-386.

10 Roe RH, Lass JH, Brown GC, Brown MM. The value-based medicine comparative effectiveness and cost-effectiveness of penetrating keratoplasty for keratoconus. Cornea 2008; 27(9): 1001-1007.

11 Koo TS, Finkelstein E, Tan D, Mehta JS. Incremental costutility analysis of deep anterior lamellar keratoplasty compared with penetrating keratoplasty for the treatment of keratoconus. Am J Ophthalmol 2011; 152(1): 40-42.

12 Yildiz EH, Cohen EJ, Virdi AS, Hammersmith KM, Laibson PR, Rapuano CJ. Quality of life in keratoconus patients after penetrating keratoplasty. Am J Ophthalmol 2010; 149(3): 416-422.

13 Kymes SM, Walline JJ, Zadnik K, Sterling J, Gordon MO. Changes in the quality-of-life of people with keratoconus. Am J Ophthalmol 2008; 145(4): 611-617.

14 O'Brart DPS, Chan E, Samaras K, Patel P, Shah SP. A randomised, prospective study to investigate the efficacy of riboflavin/ultraviolet A $(370 \mathrm{~nm})$ corneal collagen crosslinkage to halt the progression of keratoconus. $\mathrm{Br} \mathrm{J}$ Ophthalmol 2011; 95(11): 1519-1524. 
15 Fraser CG, Fogarty Y. Interpreting laboratory results. BMJ 1989; 298(6689): 1659-1660.

16 Szalai E, Berta A, Hassan Z, Módis Jr., L. Reliability and repeatability of swept-source Fourier-domain optical coherence tomography and Scheimpflug imaging in keratoconus. J Cataract Refract Surg 2012; 38(3): 485-494.

17 Wollensak G, Spoerl E, Seiler T. Riboflavin/ultraviolet-ainduced collagen crosslinking for the treatment of keratoconus. Am J Ophthalmol 2003; 135(5): 620-627.

18 Hersh PS, Greenstein SA, Fry KL. Corneal collagen crosslinking for keratoconus and corneal ectasia: one-year results. J Cataract Refract Surg 2011; 37(1): 149-160.

19 Caporossi A, Mazzotta C, Baiocchi S, Caporossi T. Longterm results of riboflavin ultraviolet a corneal collagen cross-linking for keratoconus in Italy: the Siena eye cross study. Am J Ophthalmol 2010; 149(4): 585-593.

20 Vinciguerra P, Albé E, Frueh BE, Trazza S, Epstein D. Two-year corneal cross-linking results in patients younger than 18 years with documented progressive keratoconus. AJOPHT 2012; 23: 1-7.

21 Wittig-Silva C, Whiting M, Lamoureux E, Lindsay RG, Sullivan LJ, Snibson GR. A randomized controlled trial of corneal collagen cross-linking in progressive keratoconus: preliminary results. J Refract Surg 2008; 24(7): S720-S725.

22 Koller T, Mrochen M, Seiler T. Complication and failure rates after corneal crosslinking. J Cataract Refract Surg 2009; 35(8): 1358-1362.

23 Snibson GR. Collagen cross-linking: a new treatment paradigm in corneal disease-a review. Clin Exp Ophthalmol 2010; 38(2): 141-153.

24 Spoerl E, Mrochen M, Sliney D, Trokel S, Seiler T. Safety of UVA-riboflavin cross-linking of the cornea. Cornea 2007; 26(4): 385-389.

25 Kymionis GD, Portaliou DM, Diakonis VF, Kounis GA, Panagopoulou SI, Grentzelos MA. Corneal collagen cross-linking with riboflavin and ultraviolet-A irradiation in patients with thin corneas. Am J Ophthalmol 2012; 153(1): 24-28.

26 Gokhale NS. Corneal endothelial damage after collagen cross-linking treatment. Cornea 2011; 30(12): 1495-1498.

27 Wollensak G, Spörl E, Reber F, Pillunat L, Funk R. Corneal endothelial cytotoxicity of riboflavin/UVA treatment in vitro. Ophthalmic Res 2003; 35(6): 324-328.

28 Hafezi F, Mrochen M, Iseli HP, Seiler T. Collagen crosslinking with ultraviolet-A and hypoosmolar riboflavin solution in thin corneas. J Cataract Refract Surg ASCRS and ESCRS 2009; 35(4): 621-624.

29 Raiskup F, Kißner A, Spoerl E, Pillunat LE. Hornhautvernetzung mit hypoosmolarer RiboflavinLösung beim Keratokonus mit dünner Hornhaut [Corneal cross-linking with hypo-osmolar riboflavin solution for keratoconus with thin corneas]. Ophthalmologe 2011; 108(9): 846-851.

30 Schumacher S, Oeftiger L, Mrochen M. Equivalence of biomechanical changes induced by rapid and standard corneal cross-linking, using riboflavin and ultraviolet radiation. Invest Ophthalmol Vis Sci 2011; 52(12): 9048-9052.

31 Kanellopoulos J. Long term results of a prospective randomized bilateral eye comparison trial of higher fluence, shorter duration ultraviolet A radiation, and riboflavin collagen cross linking for progressive keratoconus. Clin Ophthalmol 2012; 6: 97-101.

32 Zhang Y, Sukthankar P, Tomich JM, Conrad GW. Effect of the synthetic NC-1059 peptide on diffusion of riboflavin across an intact corneal epithelium. Invest Ophthalmol Vis Sci 2012; 53(6): 2620-2629.

33 Leccisotti A, Islam T. Transepithelial corneal collagen crosslinking in keratoconus. J Refract Surg 2010; 26(12): 942-948.

34 Filippello M, Stagni E, O’Brart D. Transepithelial corneal collagen crosslinking: bilateral study. J Cataract Refract Surg 2012; 38(2): 283-291.

35 Koppen C, Wouters K, Mathysen D, Rozema J, Tassignon MJ. Refractive and topographic results of benzalkonium chloride-assisted transepithelial crosslinking. J Cataract Refract Surg 2012; 38(6): 1000-1005.

36 Stojanovic A, Chen X, Jin N, Zhang T, Stojanovic F, Raeder S et al. Safety and efficacy of epithelium-on corneal collagen cross-linking using a multifactorial approach to achieve proper stromal riboflavin saturation. J Ophthalmol 2012; 2012: $1-8$

37 Tuft SJ, Moodaley LC, Gregory WM, Davison CR, Buckley RJ. Prognostic factors for the progression of keratoconus. Ophthalmology 1994; 101(3): 439-447.

38 Gordon MO, Steger-May K, Szczotka-Flynn L, Riley C, Joslin CE, Weissman BA et al. Baseline factors predictive of incident penetrating keratoplasty in keratoconus. Am J Ophthalmol 2006; 142(6): 923-930.

39 McMahon TT, Edrington TB, Szczotka-Flynn L, Olafsson $\mathrm{HE}$, Davis LJ, Schechtman KB et al. Longitudinal changes in corneal curvature in keratoconus. Cornea 2006; 25(3): 296-305.

40 Barnett M, Mannis MJ. Contact lenses in the management of keratoconus. Cornea 2011; 30(12): 1510-1516.

41 Ozbek Z, Cohen EJ. Use of intralimbal rigid gas-permeable lenses for pellucid marginal degeneration, keratoconus, and after penetrating keratoplasty. Eye Contact Lens 2006; 32(1): 33-36.

42 Pecego M, Barnett M, Mannis MJ, Durbin-Johnson B. Jupiter scleral lenses: the UC Davis Eye Center experience. Eye Contact Lens 2012; 38(3): 179-182.

43 Piñero DP, Alio JL, Kady El B, Coskunseven E, Morbelli H, Uceda-Montanes A et al. Refractive and aberrometric outcomes of intracorneal ring segments for keratoconus: mechanical versus femtosecond-assisted procedures. Ophthalmology 2009; 116(9): 1675-1687.

44 Kubaloglu A, Cinar Y, Sari ES, Koytak A, Ozdemir B, Ozertürk Y. Comparison of 2 intrastromal corneal ring segment models in the management of keratoconus. J Cataract Refract Surg 2010; 36(6): 978-985.

45 Rabinowitz YS, Li X, Ignacio TS, Maguen E. INTACS inserts using the femtosecond laser compared to the mechanical spreader in the treatment of keratoconus. J Refract Surg 2006; 22(8): 764-771.

46 Kubaloglu A, Sari ES, Cinar Y, Cingu K, Koytak A, Coşkun E et al. Comparison of mechanical and femtosecond laser tunnel creation for intrastromal corneal ring segment implantation in keratoconus: prospective randomized clinical trial. J Cataract Refract Surg 2010; 36(9): 1556-1561.

47 Coskunseven E, Kymionis GD, Tsiklis NS, Atun S, Arslan E, Siganos CS et al. Complications of intrastromal corneal ring segment implantation using a femtosecond laser for channel creation: a survey of 850 eyes with keratoconus. Acta Ophthalmol 2011; 89(1): 54-57.

48 Coskunseven E, Kymionis GD, Tsiklis NS, Atun S, Arslan E, Jankov MR et al. One-year results of intrastromal corneal ring segment implantation (KeraRing) using femtosecond laser in patients with keratoconus. Am J Ophthalmol 2008; 145(5): 775-779. 
49 Shabayek MH, Alio JL. Intrastromal corneal ring segment implantation by femtosecond laser for keratoconus correction. Ophthalmology 2007; 114(9): 1643-1652.

50 Haddad W, Fadlallah A, Dirani A, Rami El H, Fahd D, Khanafer D et al. Comparison of 2 types of intrastromal corneal ring segments for keratoconus. J Cartaract Refract Surg 2012; 38(7): 1214-1221.

51 Alio JL, Shabayek MH, Artola A. Intracorneal ring segments for keratoconus correction: long-term follow-up. J Cartaract Refract Surg 2006; 32(6): 978-985.

52 Coskunseven E, Jankov MR, Hafezi F, Atun S, Arslan E, Kymionis GD. Effect of treatment sequence in combined intrastromal corneal rings and corneal collagen crosslinking for keratoconus. J Cataract Refract Surg 2009; 35(12): 2084-2091.

53 Saelens IEY, Bartels MC, Bleyen I, Van Rij G. Refractive, topographic, and visual outcomes of same-day corneal cross-linking with ferrara intracorneal ring segments in patients with progressive keratoconus. Cornea 2011; 30(12): 1406-1408.

54 El-Raggal TM. Sequential versus concurrent KERARINGS insertion and corneal collagen cross-linking for keratoconus. Br J Ophthalmol 2011; 95(1): 37-41.

55 Chan CCK, Sharma M, Wachler BSB. Effect of inferiorsegment Intacs with and without C3-R on keratoconus. J Cataract Refract Surg 2007; 33(1): 75-80.

56 Ertan A, Karacal H, Kamburoğlu G. Refractive and topographic results of transepithelial cross-linking treatment in eyes with intacs. Cornea 2009; 28(7): 719-723.

57 Kato N, Toda I, Kawakita T, Sakai C, Tsubota K. Topography-guided conductive keratoplasty: treatment for advanced keratoconus. Am J Ophthalmol 2010; 150(4): 481-489.

58 Kymionis GD, Kontadakis GA, Kounis GA, Portaliou DM, Karavitaki AE, Magarakis $\mathrm{M}$ et al. Simultaneous topography-guided PRK followed by corneal collagen cross-linking for keratoconus. J Refract Surg 2009; 25(9): S807-S811.

59 Kanellopoulos AJ, Binder PS. Collagen cross-linking (CCL) with sequential topography-guided PRK: a temporizing alternative for keratoconus to penetrating keratoplasty. Cornea 2007; 26(7): 891-895.

60 Kanellopoulos AJ. Comparison of sequential vs same-day simultaneous collagen cross-linking and topographyguided PRK for treatment of keratoconus. J Refract Surg 2009; 25(9): S812-S818.

61 Alfonso JF, Palacios A, Montés-Micó R. Myopic phakic STAAR collamer posterior chamber intraocular lenses for keratoconus. J Refract Surg 2008; 24(9): 867-874.

62 Kamiya K, Shimizu K, Kobashi H, Komatsu M, Nakamura A, Nakamura Tet al. Clinical outcomes of posterior chamber toric phakic intraocular lens implantation for the correction of high myopic astigmatism in eyes with keratoconus: 6-month follow-up. Graefes Arch Clin Exp Ophthalmol 2011; 249(7): 1073-1080.

63 Izquierdo L, Henriquez MA, McCarthy M. Artiflex phakic intraocular lens implantation after corneal collagen cross-linking in keratoconic eyes. J Refract Surg 2011; 27(7): 482-487.

64 Sedaghat M, Ansari-Astaneh M-R, Zarei-Ghanavati M, Davis SW, Sikder S. Artisan iris-supported phakic IOL implantation in patients with keratoconus: a review of 16 eyes. J Refract Surg 2011; 27(7): 489-493.

65 Alfonso JF, Fernández-Vega L, Lisa C, Fernandes P, González-Méijome JM, Montés-Micó R. Collagen copolymer toric posterior chamber phakic intraocular lens in eyes with keratoconus. J Cataract Refract Surg 2010; 36(6): 906-916.

66 Kato N, Toda I, Hori-Komai Y, Sakai C, Arai H, Tsubota K. Phakic intraocular lens for keratoconus. Ophthalmology 2011; 118(3): 605-605.

67 Alfonso JF, Lisa C, Abdelhamid A, Montés-Micó R, PooLópez A, Ferrer-Blasco T. Posterior chamber phakic intraocular lenses after penetrating keratoplasty. J Cataract Refract Surg 2009; 35(7): 1166-1173.

68 Kohnen T, Kook D, Morral M, Güell JL. Phakic intraocular lenses: part 2: results and complications. J Cataract Refract Surg 2010; 36(12): 2168-2194.

69 Pesando PM, Ghiringhello MP, Di Meglio G, Fanton G. Posterior chamber phakic intraocular lens (ICL) for hyperopia: ten-year follow-up. J Cataract Refract Surg 2007; 33(9): 1579-1584.

70 Edelhauser HF, Sanders DR, Azar R, Lamielle H. ICL in Treatment of Myopia Study Group. Corneal endothelial assessment after ICL implantation. J Cataract Refract Surg 2004; 30(3): 576-583.

71 Alfonso JF, Lisa C, Fernández-Vega L, Madrid-Costa D, PooLópez A, Montés-Micó R. Intrastromal corneal ring segments and posterior chamber phakic intraocular lens implantation for keratoconus correction. J Cataract Refract Surg 2011; 37(4): 706-713.

72 Moshirfar M, Fenzl CR, Meyer JJ, Neuffer MC, Espandar L, Mifflin MD. Simultaneous and sequential implantation of Intacs and Verisyse phakic intraocular lens for refractive improvement in keratectasia. Cornea 2011; 30(2): 158-163.

73 Cakir H, Utine CA. Combined Kerarings and Artisan/Artiflex IOLs in keratectasia. J Refract Surg 2010; 27(2): 119-126.

74 El-Raggal TM, Abdel Fattah AA. Sequential Intacs and Verisyse phakic intraocular lens for refractive improvement in keratoconic eyes. J Cataract Refract Surg 2007; 33(6): 966-970.

75 Huang D, Schallhorn SC, Sugar A, Farjo AA, Majmudar PA, Trattler WB et al. Phakic intraocular lens implantation for the correction of myopia. Ophthalmology 2009; 116(11): 2244-2258.

76 Raiskup-Wolf F, Hoyer A, Spoerl E, Pillunat LE. Collagen crosslinking with riboflavin and ultraviolet-A light in keratoconus: long-term results. J Cataract Refract Surg 2008; 34(5): 796-801. 